\title{
CONTROL DE SAINILLO (Dieffenbachia oerstedii Schott) EN BANANO ${ }^{1}$
}

\author{
Steven Brenes-Prendas ${ }^{2}$, Renán Agüero-Alvarado ${ }^{3}$, Lili Hofmann ${ }^{4}$
}

\section{RESUMEN}

Control de sainillo (Dieffenbachia oerstedii Schott) en banano. De junio a diciembre del 2006, en la finca Limofrut C, ubicada en el cantón de Matina, Limón, se realizaron tres experimentos, con el fin de evaluar diferentes alternativas de control de sainillo. En el primer experimento se evaluó un testigo a libre crecimiento de arvenses, la deshierba manual y once tratamientos químicos. En el segundo experimento, se evaluaron los tratamientos triclopir + carfentrazone; triclopir + carfentrazone + sulfato de amonio; triclopir + glifosato + carfentrazone + sulfato de amonio y metsulfuron metil + carfentrazone, ya que mostraron el mejor control de sainillo durante el primer experimento; además, se evaluó el efecto de "untarlos" sobre hojas, tallos, o sobre ambos. En el tercer experimento, se evaluaron tratamientos con registro EPA en banano, a saber glifosato; glifosato + sulfato de amonio y glifosato + carfentrazone. Los tratamientos con metsulfuron metil + carfentrazone y glifosato + carfentrazone dieron el mayor control de sainillo. Este último tratamiento ya se validó con éxito, en áreas comerciales. A pesar de que el herbicida triclopir fue untado sobre la maleza, con frecuencia la lluvia depositó suficiente herbicida sobre plantas de banano las cuales mostraron una severa toxicidad; por ello se excluyó este herbicida de futuros estudios. Se encontraron bacterias de los géneros Pseudomonas y Erwinia asociadas a la sintomatología en los tratamientos con los herbicidas; se discute su potencial como agentes de control biológico.

Palabras clave: Arvenses, recalcitrantes, metsulfuron metil, carfentrazone, glifosato.

\begin{abstract}
Sainillo (Dieffenbachia oerstedii Schott) control in bananas. Three experiments were conducted to evaluate the control of sainillo, from June to December of 2006 in Limofrut $\mathrm{C}$ farm located in Matina, Limón. Experiment one consisted of exploratory treatments that included an untreated check, a manual control check and eleven chemical treatments. Experiment two served to compare with greater detail, the best treatments observed during experiment one; tryclopir + carfentrazone; tryclopir + carfentrazone + ammonium sulphate; tryclopir + glyphosate + carfentrazone + ammonium sulphate and metsulfuron methyl + carfentrazone; these treatments were smeared on leaves, stems and both. Experiment three included only herbicides registered by EPA for use in bananas; glyphosate; glyphosate + ammonium sulphate; glyphosate + carfentrazone. Metsulfuron methyl + carfentrazone and glyphosate + carfentrazone showed the best control of sainillo. Glyphosate + carfentrazone rendered satisfactory control of sainillo in commercial validation plots. Even though treatments with tryclopir were smeared on the weed plants, enough herbicide carried by rain, often reached banana plants, causing severed damage and thus this herbicide was dropped from further testing. Pseudomonas spp and Erwinia spp were found as important contributing factors in the death of weed plants, in the plots treated with herbicides. Their potencial for biological control of this weed is discussed.
\end{abstract}

Key words: Weeds, recalcitrants, metsulfuron methyl, carfentrazone, glyphosate.

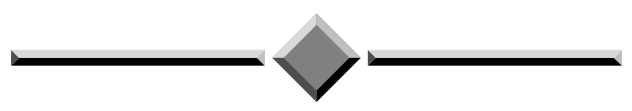

1 Recibido: 11 de julio, 2007. Aceptado: 26 de agosto, 2008. Artículo que forma parte de la tesis de maestría del primer autor.

2 Estudiante del Sistema de Posgrado en Ciencias Agrícolas y Recursos Naturales de la Universidad de Costa Rica, San José, Costa Rica. sbrenesp@gmail.com

3 Laboratorio de Arvenses (LAR) del Centro de Investigaciones en Protección de Cultivos (CIPROC), Universidad de Costa Rica, San José, Costa Rica.ragueroster@gmail.com

4 Laboratorio de técnicas moleculares del CIPROC, Universidad de Costa Rica. hofmannl@agro.ucr.ac.cr 


\section{INTRODUCCIÓN}

En la composición florística asociada al agroecosistema bananero existe un grupo de arvenses que escapan a los tratamientos de control que se usan en la actualidad. Debido a sus características de difícil manejo, su potencial de causar alergias a los trabajadores, dificultar las labores de campo (Rodríguez y Agüero 2000), y la competencia que ejercen sobre el cultivo, son consideradas como arvenses recalcitrantes.

Entre las arvenses recalcitrantes se encuentra el sainillo (Dieffenbachia oerstedii), la cual es una planta herbácea que se desarrolla en ambientes de umbría y alta humedad (Hammel 2005, Arias 1998, Croat 1991), características típicas del agroecosistema bananero en la zona Atlántica de Costa Rica. Esta planta, en su estado adulto, alcanza hasta $1,5 \mathrm{~m}$ de altura; posee un tallo suculento con numerosos entrenudos que al doblarse y entrar en contacto con el suelo tiende a desarrollar raíces, siendo éste, su principal modo de reproducción (Nilsson et al. 2005), por lo que no se recomienda su control mediante chapeas.

Una de las características que hacen del sainillo una arvense problemática, es su savia lechosa con altos contenidos de cristales de oxalato de calcio conocidos como rafides, que al entrar en contacto con la piel, ojos o ser injeridos, causan irritaciones, alergias, pérdida momentánea de la visión, asfixia e incluso esterilidad parcial o total (Nilsson et al. 2005, Egerer 2005, Matysik 2005, Kissman 2000, Prychid y Rudall 1999, Zettler et al. 1975). Se han reportado trabajadores bananeros que han perdido la visión temporalmente al caer savia en sus ojos, y han sido incapacitados por varios meses.

El sainillo crece en forma de parches densos (Croat 1991) dentro de las plantaciones, lo que facilita el contacto del trabajador con la planta; además, dificulta las labores de fertilización, aplicación de agroquímicos y cosecha de la fruta. Además por la cercanía de estos parches a la planta de banano, es probable que exista competencia con el cultivo por nutrientes y espacio.

La presente investigación muestra las alternativas que se desarrollaron para el control del sainillo, en un agroecosistema bananero.

\section{MATERIALES Y MÉTODOS}

\section{Ubicación}

El estudio se realizó en los cables cinco y seis de la finca Limofrut C, en Matina, provincia de Limón, en el período de junio a diciembre del 2006. Según la clasificación de zonas de vida de Holdridge (1979), el lugar corresponde a la formación de bosque tropical húmedo, con una precipitación anual que fluctúa entre los 3.500 a $4.000 \mathrm{~mm}$. La temperatura anual promedio es de $25^{\circ} \mathrm{C}$ y la humedad promedio del $85 \%$ (Herrera 1985).

Los productos utilizados en esta investigación fueron carfentrazone, glifosato, metsulfuron metil, triclopir y Nu film 17 P, este último como coadyuvante.

La fase experimental se dividió en tres experimentos que se detallan a continuación. Todos ellos fueron realizados en plantaciones de banano variedad Grande Naine.

\section{Experimento 1}

Este primer experimento, realizado del 8 de junio al 14 de setiembre del 2006, consistió en evaluar diferentes tratamientos químicos para el control de sainillo. Los tratamientos evaluados se presentan en el Cuadro 1. El diseño experimental utilizado fue un irrestricto al azar con tres repeticiones; la unidad experimental consistió de una parcela de $1 \mathrm{~m}^{2}$.

En el caso de los tratamientos tres y cuatro, éstos fueron asperjados sobre la maleza con una bomba de espalda de 16 litros, con una boquilla Tee Jet 8002 . Los demás tratamientos fueron untados sobre la parte aérea del sainillo con un mechero Microwipe (Micro Sprayers LTD, Reino Unido), con un volumen de mezcla de $400 \mathrm{ml}$ para cada tratamiento. Se registró el volumen consumido en cada uno, luego de finalizar el untado.

Las variables evaluadas fueron: porcentaje de cobertura, altura, número de tallos totales y número de tallos de la maleza afectados por los tratamientos. 
Cuadro 1. Tratamientos utilizados en el primer experimento, para el control de sainillo (Dieffenbachia oerstedii Schott) en banano. Finca Limofrut C. Matina, Limón, Costa Rica. 2006.

\begin{tabular}{|c|c|c|}
\hline \# Trat & Descripción de tratamientos & Dosis \\
\hline 1 & Testigo a libre crecimiento de la maleza & \\
\hline 2 & Deshierba manual a ras de suelo & \\
\hline 3 & Glifosato + carfentrazone + coadyuvante & $1,080 \mathrm{~kg}$ e.a. $/ \mathrm{ha}+0,048 \mathrm{~kg}$ i.a. $/ \mathrm{ha}+0,25 \% \mathrm{v} / \mathrm{v}$ \\
\hline 4 & Glifosato + carfentrazone + sulfato de amonio + coadyuvante & $1,080 \mathrm{~kg}$ e.a. $/ \mathrm{ha}+0,048 \mathrm{~kg}$ i.a. $/ \mathrm{ha}+4 \mathrm{~kg} / \mathrm{ha}+0,25 \% \mathrm{v} / \mathrm{v}$ \\
\hline 5 & Triclopir + coadyuvante & $33: 0,5 \%$ vpc* / 66,5 \% v agua** \\
\hline 6 & Triclopir + carfentrazone + coadyuvante & $25: 8: 0,5 \%$ vpc / $66,5 \%$ v agua \\
\hline 7 & Triclopir + carfentrazone + sulfato de amonio + coadyuvante & $20: 8: 10: 0,5 \%$ vpc / $61,5 \%$ v agua \\
\hline 8 & Triclopir + glifosato + sulfato de amonio + coadyuvante & $16: 16: 10: 0,5 \%$ vpc / $57,5 \%$ v agua \\
\hline 9 & Hidroxal $* * *+$ coadyuvante & $33: 0,5 \%$ vpc / $66,5 \%$ v agua \\
\hline 10 & Hidroxal + carfentrazone + coadyuvante & $25: 8: 0,5 \%$ vpc / 66,5 \% v agua \\
\hline 11 & Metsulfuron metil $* * * *+$ coadyuvante & $18,75: 0,5 \% \mathrm{vpc} / 80,75 \% \mathrm{v}$ agua \\
\hline 12 & Metsulfuron metil + carfentrazone + coadyuvante & $18,75: 8: 0,5 \%$ vpc $/ 72,75 \%$ v agua \\
\hline 13 & $\begin{array}{l}\text { Metsulfuron metil + carfentrazone + sulfato de amonio + } \\
\text { coadyuvante }\end{array}$ & $18,75: 8: 10: 0,5 \%$ vpc / $62,75 \%$ v agua \\
\hline
\end{tabular}

*\% vpc: porcentaje del volumen total que corresponde a cada producto comercial

** \% v agua: porcentaje del volumen total que corresponde al agua (no dura, $\mathrm{pH}$ neutro).

*** hidroxal: $5,4 \% \mathrm{p} / \mathrm{v}$ de hidróxido de aluminio $+1,8 \mathrm{p} / \mathrm{v}$ de hidróxido de magnesio

**** En los tratamientos 11, 12 y 13 el volumen de metsulfuron metil es referido a una solución madre de 10 g de producto comercial al $75 \%$, en $300 \mathrm{ml}$ de agua.

\section{Experimento 2}

El segundo experimento, realizado del 20 de julio al 4 de noviembre del 2006, se seleccionaron los tratamientos del primer experimento, que mostraron mayor control sobre el sainillo y untarlos en diferentes partes de la arvense, a saber: a) sólo en las hojas, b) sólo en el tallo y c) en hojas y tallos. Los tratamientos evaluados se resumen en el Cuadro 2. Estos fueron untados sobre la maleza con el mismo equipo utilizado para untar los tratamientos del primer experimento.

El diseño experimental utilizado fue un irrestricto al azar con un arreglo factorial $4 \times 3$ (cuatro tratamientos de control y tres partes de la planta donde se untaron esos tratamientos), y se usaron tres repeticiones para cada tratamiento. La unidad experimental tuvo una área de $1 \mathrm{~m}^{2}$.

En el sainillo se evaluó el porcentaje de cobertura, altura, número de tallos totales y número de tallos afectados por los tratamientos.

\section{Experimento 3}

En este experimento se evaluaron alternativas de control químico del sainillo, con registro EPA para el cultivo de banano (Cuadro 3), del 9 al 24 de agosto del 2006. Estos tratamientos fueron untados sobre toda la parte aérea de la arvense, con el mismo equipo utilizado para untar los tratamientos del primer y segundo experimento.

El diseño utilizado fue un irrestricto al azar con cuatro repeticiones. La unidad experimental consistió de una parcela de $1 \mathrm{~m}^{2}$ de área. Se evaluó en el sainillo el porcentaje de cobertura, altura, número de tallos totales y número de tallos afectados por los tratamientos.

En las variables de altura de plantas de sainillo y rebrotes no se pudo detectar diferencias significativas entre los tratamientos en esta única evaluación (Cuadros 18 y 19). Ocurrió interferencia de algunas prácticas realizadas por la finca sobre las unidades experimentales. Una recaba de los drenajes cercanos a 
Cuadro 2. Tratamientos evaluados en el segundo experimento, para el control de sainillo (Dieffenbachia oerstedii Schott) en banano. Finca Limofrut C, Matina, Limón, Costa Rica. 2006.

\begin{tabular}{|c|c|c|}
\hline \# Trat & Descripción de tratamientos & Dosis \\
\hline 1 & Triclopir + carfentrazone + coadyuvante $/$ hojas + tallos & $25: 8: 0,5 \%$ vpc $* / 66,5 \%$ v agua** $^{* *}$ \\
\hline 2 & Triclopir + carfentrazone + coadyuvante/ sólo a hojas & $25: 8: 0,5 \%$ vpc / $66,5 \%$ v agua \\
\hline 3 & Triclopir + carfentrazone + coadyuvante/ sólo a tallos & $25: 8: 0,5 \% \mathrm{vpc} / 66,5 \% \mathrm{v}$ agua \\
\hline 4 & Triclopir + carfentrazone + sulfato de amonio + coadyuvante $/$ hojas + tallos & $20: 8: 10: 0,5 \%$ vpc / $61,5 \%$ v agua \\
\hline 5 & Triclopir + carfentrazone + sulfato de amonio + coadyuvante / sólo a hojas & $20: 8: 10: 0,5 \%$ vpc / $61,5 \%$ v agua \\
\hline 6 & Triclopir + carfentrazone + sulfato de amonio + coadyuvante / sólo a tallos & $20: 8: 10: 0,5 \%$ vpc / $61,5 \%$ v agua \\
\hline 7 & $\begin{array}{l}\text { Triclopir + glifosato + carfentrazone + sulfato de amonio + coadyuvante } / \text { hojas } \\
+ \text { tallos }\end{array}$ & $16: 16: 10: 0,5 \%$ vpc / $57,5 \%$ v agua \\
\hline 8 & $\begin{array}{l}\text { Triclopir + glifosato + carfentrazone + sulfato de amonio + coadyuvante / sólo } \\
\text { a hojas }\end{array}$ & $16: 16: 10: 0,5 \%$ vpc / $57,5 \%$ v agua \\
\hline 9 & $\begin{array}{l}\text { Triclopir + glifosato + carfentrazone + sulfato de amonio + coadyuvante / sólo } \\
\text { a tallos }\end{array}$ & $16: 16: 10: 0,5 \%$ vpc / $57,5 \%$ v agua \\
\hline 10 & Metsulfuron metil $* * *+$ carfentrazone + coadyuvante $/$ hojas + tallos & $18,75: 8: 0,5 \%$ vpc $/ 72,75 \%$ v agua \\
\hline 11 & Metsulfuron metil**** carfentrazone + coadyuvante / sólo a hojas & $18,75: 8: 0,5 \%$ vpc $/ 72,75 \%$ v agua \\
\hline 12 & Metsulfuron metil ${ }^{* * *}+$ carfentrazone + coadyuvante / sólo a tallos & $18,75: 8: 0,5 \%$ vpc / 72,75\% v agua \\
\hline
\end{tabular}

*\% vpc: porcentaje del volumen total que corresponde a cada producto comercial

** \% v agua: porcentaje del volumen total que corresponde al agua (no dura, $\mathrm{pH}$ neutro).

*** En los tratamientos 10,11 y 12 el volumen de metsulfuron metil es referido a una solución madre de 10 g de producto comercial al $75 \%$, en $300 \mathrm{ml}$ de agua.

Cuadro 3. Tratamientos evaluados en el tercer experimento, para el control de sainillo (Dieffenbachia oerstedii Schott) en banano. Finca Limofrut C. Matina, Limón, Costa Rica. 2006.

\begin{tabular}{|c|c|c|}
\hline $\begin{array}{c}\# \\
\text { Trat }\end{array}$ & $\begin{array}{l}\text { Descripción de } \\
\text { tratamientos }\end{array}$ & Dosis \\
\hline 1 & Glifosato + coadyuvante & $\begin{array}{l}33: 1 \% \text { vpc* / } 66 \% \text { v } \\
\text { agua** }\end{array}$ \\
\hline 2 & $\begin{array}{l}\text { Glifosato + sulfato de } \\
\text { amonio + coadyuvante }\end{array}$ & $\begin{array}{l}33: 10: 1 \% \text { vpc / } 56 \% \\
\text { v agua }\end{array}$ \\
\hline 3 & $\begin{array}{l}\text { Glifosato + carfentrazone } \\
(\text { dosis } 1)+\text { coadyuvante }\end{array}$ & $\begin{array}{l}33: 4: 1 / 62 \% \mathrm{vpc} / \mathrm{v} \\
\text { agua }\end{array}$ \\
\hline 4 & $\begin{array}{l}\text { Glifosato + carfentrazone } \\
(\text { dosis } 2)+\text { coadyuvante }\end{array}$ & $\begin{array}{l}33: 8: 1 / 58 \% \mathrm{vpc} / \mathrm{v} \\
\text { agua }\end{array}$ \\
\hline 5 & $\begin{array}{l}\text { Glifosato + carfentrazone } \\
(\text { dosis } 3)+\text { coadyuvante }\end{array}$ & $\begin{array}{l}33: 16: 1 / 50 \% \mathrm{vpc} / \mathrm{v} \\
\text { agua }\end{array}$ \\
\hline
\end{tabular}

$* \%$ vpc: porcentaje del volumen total que corresponde a cada producto comercial.

** \% v agua: porcentaje del volumen total que corresponde al agua (no dura, $\mathrm{pH}$ neutro). las unidades experimentales, así como la aplicación de cal- dolomita, dañaron parcialmente dichas unidades, por lo que sólo se cuenta con una evaluación de este estudio.

Los datos obtenidos en los tres estudios se analizaron mediante un análisis de varianza y las medias se compararon con la prueba de rangos múltiples DMS protegida de Fischer, al 5\% de probabilidad.

Se seleccionó el tratamiento de glifosato + carfentazone (33:8:1\% vpc / 58\% v agua) del tercer experimento, para realizar una prueba de validación en áreas de plantación comercial de banano, del 3 de noviembre del 2006 al 20 de febrero del 2007. Se tomaron seis puntos al azar de las áreas aplicadas, en las cuales se evaluaron las variables de porcentaje de cobertura, altura, número de tallos totales y número de tallos afectados por los tratamientos, utilizando como unidad experimental una parcela de $1 \mathrm{~m}^{2}$. 


\section{RESULTADOS Y DISCUSIÓN}

\section{Experimento 1}

No se presentaron diferencias significativas entre los tratamientos en la primera evaluación, en las variables de porcentaje de cobertura y altura de plantas (Cuadros 4 y 6), lo que sugiere una adecuada homogeneidad poblacional, deseable al inicio. Para la segunda evaluación, posterior a la aplicación de los tratamientos, se observó que los tratamientos de triclopir + carfentrazone + surfactante (T6), triclopir +

Cuadro 4. Cobertura del suelo por sainillo (Dieffenbachia oerstedii Schott) en banano en cinco épocas. Finca Limofrut C, Matina, Limón, Costa Rica. 2006.

\begin{tabular}{llllll}
\hline \multicolumn{1}{c}{ Tratamientos } & \multicolumn{5}{c}{ Épocas de evaluación } \\
\cline { 2 - 6 } & Jun 6** & Jun 24* & Jul 6* & Jul 21* & Set 14* \\
\hline Testigo libre crec arvense & $61,6 \mathrm{a}$ & $71,4 \mathrm{a}$ & $72,0 \mathrm{a}$ & $73,8 \mathrm{a}$ & $74,8 \mathrm{a}$ \\
Deshierba manual & $67,5 \mathrm{a}$ & $4,9 \mathrm{f}$ & $12,9 \mathrm{c}$ & $16,8 \mathrm{~d}$ & $46,9 \mathrm{bc}$ \\
gli + carf + coad & $67,4 \mathrm{a}$ & $57,7 \mathrm{ab}$ & $59,2 \mathrm{a}$ & $56,0 \mathrm{~b}$ & $59,7 \mathrm{~b}$ \\
gli + carf + sulftato + coad & $73,5 \mathrm{a}$ & $52,1 \mathrm{bc}$ & $36,9 \mathrm{~b}$ & $34,2 \mathrm{c}$ & $38,0 \mathrm{~cd}$ \\
tri + coad & $62,5 \mathrm{a}$ & $38,1 \mathrm{~cd}$ & $31,3 \mathrm{~b}$ & $17,5 \mathrm{~d}$ & $29,3 \mathrm{de}$ \\
tri + carf + coad & $64,8 \mathrm{a}$ & $20,8 \mathrm{e}$ & $14,8 \mathrm{c}$ & $13,5 \mathrm{~d}$ & $22,5 \mathrm{ef}$ \\
tri + carf + sulfato + coad & $79,7 \mathrm{a}$ & $29,5 \mathrm{de}$ & $16,2 \mathrm{c}$ & $10,4 \mathrm{~d}$ & $19,3 \mathrm{ef}$ \\
tri + gli + sulfato + coad & $70,9 \mathrm{a}$ & $27,5 \mathrm{de}$ & $16,2 \mathrm{c}$ & $12,2 \mathrm{~d}$ & $22,7 \mathrm{ef}$ \\
hidroxal + coad & $65,0 \mathrm{a}$ & $65 \mathrm{ab}$ & $66,6 \mathrm{a}$ & $66,1 \mathrm{ab}$ & $59 \mathrm{~b}$ \\
hidro + carf + coad & $80,8 \mathrm{a}$ & $59,7 \mathrm{ab}$ & $59,3 \mathrm{a}$ & $69,2 \mathrm{a}$ & $81,4 \mathrm{a}$ \\
metsul+ coad & $74,7 \mathrm{a}$ & $46,0 \mathrm{bc}$ & $25,5 \mathrm{bc}$ & $14,9 \mathrm{~d}$ & $9,3 \mathrm{f}$ \\
metsul + carf + coad & $69,6 \mathrm{a}$ & $38,2 \mathrm{~cd}$ & $16,2 \mathrm{c}$ & $10,4 \mathrm{~d}$ & $6,2 \mathrm{f}$ \\
metsul + carf + sulfato+ sur & $71,3 \mathrm{a}$ & $45,9 \mathrm{bc}$ & $31,3 \mathrm{~b}$ & $21,9 \mathrm{~d}$ & $11,7 \mathrm{f}$ \\
\hline
\end{tabular}

Medias con la misma letra en la misma columna no difieren significativamente, según DMS, $\mathrm{p}<=0,05$.

* Datos transformados a arcoseno raíz x.

**1= el día antes de la aplicación de los tratamientos.

Cuadro 5. Porcentaje de tallos sintomáticos de sainillo (Dieffenbachia oerstedii Schott) en banano, en cuatro épocas. Finca Limofrut C, Matina, Limón, Costa Rica. 2006.

\begin{tabular}{lllll}
\hline \multicolumn{1}{c}{ Tratamientos } & \multicolumn{4}{c}{ Épocas de evaluación } \\
\cline { 2 - 4 } & Jun 24* & Jul 6* & Jul 21* & Set 14* \\
\hline Testigo libre crec arvense & $4,6 \mathrm{~d}$ & $4,6 \mathrm{c}$ & $4,6 \mathrm{c}$ & $4,6 \mathrm{~d}$ \\
Deshierba manual & $4,6 \mathrm{~d}$ & $4,6 \mathrm{c}$ & $4,6 \mathrm{c}$ & $4,6 \mathrm{~d}$ \\
gli + carf + coad & $4,6 \mathrm{~d}$ & $13,7 \mathrm{c}$ & $9,1 \mathrm{c}$ & $4,6 \mathrm{~d}$ \\
gli + carf + sulftato + coad & $45,3 \mathrm{bc}$ & $43,1 \mathrm{~b}$ & $37,4 \mathrm{bc}$ & $37,7 \mathrm{c}$ \\
tri + coad & $85,4 \mathrm{a}$ & $85,4 \mathrm{a}$ & $66 \mathrm{ab}$ & $48,9 \mathrm{bc}$ \\
tri + carf + coad & $59,4 \mathrm{ab}$ & $80,5 \mathrm{a}$ & $85,4 \mathrm{a}$ & $51,8 \mathrm{bc}$ \\
tri + carf + sulfato + coad & $78,9 \mathrm{ab}$ & $85,4 \mathrm{a}$ & $68,7 \mathrm{ab}$ & $50,0 \mathrm{bc}$ \\
tri + gli + sulfato + coad & $80,5 \mathrm{ab}$ & $85,4 \mathrm{a}$ & $85,4 \mathrm{a}$ & $53,9 \mathrm{~b}$ \\
hidroxal + coad & $4,6 \mathrm{~d}$ & $7,5 \mathrm{c}$ & $4,6 \mathrm{c}$ & $4,6 \mathrm{~d}$ \\
hidro + carf + coad & $16,1 \mathrm{~cd}$ & $16,7 \mathrm{c}$ & $10,3 \mathrm{c}$ & $4,6 \mathrm{~d}$ \\
metsul+ coad & $66,7 \mathrm{ab}$ & $85,4 \mathrm{a}$ & $58,5 \mathrm{ab}$ & $73,9 \mathrm{a}$ \\
metsul + carf + coad & $85,4 \mathrm{a}$ & $82,3 \mathrm{a}$ & $58,5 \mathrm{ab}$ & $85,4 \mathrm{a}$ \\
metsul + carf + sulfato + coad & $49,6 \mathrm{a}$ & $74,5 \mathrm{a}$ & $81 \mathrm{a}$ & $78,3 \mathrm{a}$ \\
\hline
\end{tabular}

Medias con la misma letra en la misma columna no difieren significativamente, según DMS, $\mathrm{p}<=0,05$,

* Datos transformados a arcoseno raíz x. 
Cuadro 6. Altura (cm) de plantas de sainillo (Dieffenbachia oerstedii Schott) en banano, en cinco épocas. Finca Limofrut C, Matina, Limón, Costa Rica. 2006.

\begin{tabular}{llllll}
\hline \multirow{2}{*}{ Tratamientos } & \multicolumn{5}{c}{ Épocas de evaluación } \\
\cline { 2 - 5 } & Jun 6 & Jun 24 & Jul 6 & Jul 21 & Set 14 \\
\hline Testigo libre crec arvense & $100 \mathrm{a}$ & $105,7 \mathrm{a}$ & $74,3 \mathrm{ab}$ & $77,3 \mathrm{a}$ & $95,7 \mathrm{a}$ \\
Deshierba manual & $100 \mathrm{a}$ & $3,3 \mathrm{~d}$ & $11,7 \mathrm{e}$ & $13,3 \mathrm{c}$ & $33 \mathrm{c}$ \\
gli + carf + coad & $93,3 \mathrm{a}$ & $68,3 \mathrm{~b}$ & $52,3 \mathrm{bc}$ & $48 \mathrm{~b}$ & $55,7 \mathrm{~b}$ \\
gli + carf + sulftato + coad & $105 \mathrm{a}$ & $68 \mathrm{~b}$ & $43 \mathrm{~cd}$ & $47,3 \mathrm{~b}$ & $40 \mathrm{bc}$ \\
tri + coad & $110,7 \mathrm{a}$ & $24 \mathrm{~cd}$ & $22 \mathrm{de}$ & $18 \mathrm{c}$ & $21,7 \mathrm{~cd}$ \\
tri + carf + coad & $107,7 \mathrm{a}$ & $34 \mathrm{c}$ & $14 \mathrm{de}$ & $15 \mathrm{c}$ & $24,3 \mathrm{~cd}$ \\
tri + carf + sulfato + coad & $116,3 \mathrm{a}$ & $26,3 \mathrm{c}$ & $16,3 \mathrm{de}$ & $10,7 \mathrm{c}$ & $9 \mathrm{~d}$ \\
tri + gli + sulfato + coad & $99 \mathrm{a}$ & $30 \mathrm{c}$ & $18 \mathrm{de}$ & $14,3 \mathrm{c}$ & $21,3 \mathrm{~cd}$ \\
hidroxal + coad & $120 \mathrm{a}$ & $96,7 \mathrm{a}$ & $72,7 \mathrm{ab}$ & $74 \mathrm{a}$ & $81,3 \mathrm{a}$ \\
hidro + carf + coad & $122,3 \mathrm{a}$ & $96,7 \mathrm{a}$ & $75,3 \mathrm{a}$ & $77,7 \mathrm{a}$ & $99 \mathrm{a}$ \\
metsul+ coad & $105 \mathrm{a}$ & $57,7 \mathrm{~b}$ & $25 \mathrm{de}$ & $13,7 \mathrm{c}$ & $5,7 \mathrm{~d}$ \\
metsul + carf + coad & $125 \mathrm{a}$ & $56,7 \mathrm{~b}$ & $20,3 \mathrm{de}$ & $14,3 \mathrm{c}$ & $4 \mathrm{~d}$ \\
metsul + carf + sulfato + coad & $131,7 \mathrm{a}$ & $66,7 \mathrm{~b}$ & $34,7 \mathrm{~cd}$ & $24,7 \mathrm{c}$ & $7 \mathrm{~d}$ \\
\hline
\end{tabular}

Medias con la misma letra en la misma columna no difieren significativamente, según DMS, $\mathrm{p}<=0,05$.

carfentrazone + sulfato de amonio + surfactante (T7) y triclopir + glifosato + sulfato de amonio + surfactante (T8) mostraron los menores porcentajes de cobertura de sainillo, así como la menor altura de la maleza y el mayor número de tallos sintomáticos (Cuadros 5 y 6). Este resultado se repitió en la tercera evaluación (Cuadro 4). Sin embargo, en la cuarta y quinta evaluación se observó una recuperación de la cobertura, altura y número de tallos, debido al rebrote de los tallos no controlados (Cuadro 7).

En la cuarta evaluación, los tratamientos de metsulfuron metil + surfactante (T11), metsulfuron metil + carfentrazone + surfactante (T12) y metsulfuron metil + carfentrazone + sulfato de amonio + surfactante (T13), igualaron a los tratamientos con triclopir (T6,

Cuadro 7. Rebrote de plantas de sainillo (Dieffenbachia oerstedii Schott) por $\mathrm{m}^{2}$ en banano en tres épocas. Finca Limofrut C, Matina, Limón, Costa Rica. 2006.

\begin{tabular}{lccc}
\hline \multicolumn{1}{c}{ Tratamientos } & \multicolumn{3}{c}{ Épocas de evaluación } \\
\cline { 2 - 3 } & Jul 6* $^{*}$ & Jul 21* & Set 14* \\
\hline Testigo libre crec. arvense & $1,43 \mathrm{a}$ & $1,1 \mathrm{bc}$ & $1,99 \mathrm{bc}$ \\
Deshierba manual & $2,23 \mathrm{a}$ & $3,24 \mathrm{a}$ & $4,8 \mathrm{a}$ \\
gli + carf + coad & $1,56 \mathrm{a}$ & $2,18 \mathrm{ab}$ & $2,94 \mathrm{~b}$ \\
gli + carf + sulftato + coad & $1,35 \mathrm{a}$ & $1,47 \mathrm{bc}$ & $1,68 \mathrm{~cd}$ \\
tri + coad & $1,35 \mathrm{a}$ & $1,29 \mathrm{bc}$ & $2,85 \mathrm{bc}$ \\
tri + carf + coad & $1,35 \mathrm{a}$ & $1,57 \mathrm{bc}$ & $2,7 \mathrm{bc}$ \\
tri + carf + sulfato + coad & $1,17 \mathrm{a}$ & $1,1 \mathrm{bc}$ & $2,49 \mathrm{bc}$ \\
tri + gli + sulfato + coad & $1,1 \mathrm{a}$ & $1,17 \mathrm{bc}$ & $2,56 \mathrm{bc}$ \\
hidroxal + coad & $0,71 \mathrm{a}$ & $1,84 \mathrm{bc}$ & $2,62 \mathrm{bc}$ \\
hidro + carf + coad & $2,03 \mathrm{a}$ & $1,43 \mathrm{c}$ & $3,05 \mathrm{~b}$ \\
metsul + coad & $1,05 \mathrm{a}$ & $0,71 \mathrm{c}$ & $1 \mathrm{~cd}$ \\
metsul + carf + coad & $0,88 \mathrm{a}$ & $0,88 \mathrm{c}$ & $0,71 \mathrm{~d}$ \\
metsul + carf + sulfato + coad & $0,71 \mathrm{a}$ & $0,88 \mathrm{c}$ & $1,05 \mathrm{~cd}$ \\
\hline
\end{tabular}

Medias con la misma letra en la misma columna no difieren significativamente, según DMS, $\mathrm{p}<=0,05$.

* Datos transformados a raíz $\mathrm{x}+0,5$. 
T7, T8 y T9) en la menor cobertura de sainillo, así como en altura de la maleza y tallos sintomáticos. Ya en la quinta evaluación los tratamientos que incluían al herbicida metsulfuron (T11, T12 y T13) obtuvieron los menores valores de cobertura y altura de la arvense, y los mayores porcentajes de tallos sintomáticos, en relación a los otros tratamientos evaluados; además, presentaron la menor cantidad de rebrotes a lo largo del experimento (Cuadro 7).

Si bien el efecto del metsulfuron fue más lento en reducir la cobertura y la altura del sainillo, ejerció un control de esta arvense más prolongado. Además, inhibió en mayor medida su capacidad de rebrote, para un mejor control (Cuadro 7).

Los tratamientos hidroxal + surfactante (T9) e hidroxal + carfentrazone + surfactante (T10) no difirieron del testigo a libre crecimiento de la arvense, a lo largo de todo el estudio. Este tratamiento fue evaluado, ya que en esfuerzos anteriores por controlar al sainillo, por parte de los trabajadores de la finca, se indicó que este producto ejercía un significativo control sobre la maleza. Luego del presente estudio donde no presentó un control satisfactorio, se descartó como alternativa de control.

El tratamiento de deshierba manual a ras del suelo (T2), permitió evaluar la capacidad de recuperación del sainillo, cuando éste es controlado mediante chapeas. En el caso de la variable porcentaje de cobertura (Cuadro 4), se observó una recuperación paulatina, hasta alcanzar un $69,6 \%$ de cobertura con respecto a la evaluación inicial en un período de 100 días aproximadamente después de la chapea. Nótese que ésta fue una chapea a ras del suelo, con extracción de toda la biomasa cortada. La altura del sainillo, se logró recuperar un 33\% en ese mismo período (Cuadro 6). $\mathrm{Al}$ analizar rebrotes $/ \mathrm{m}^{2}$, el tratamiento de deshierba manual presentó el mayor número de rebrotes en comparación con los otros tratamientos (Cuadro 7). Por los datos antes expuestos es que la chapea o arranque manual del sainillo no es recomendable como alternativa de control, ya que estas prácticas reactivan el crecimiento del sainillo, además de dispersarlo dentro del agroecosistema bananero.

Para los Cuadros del 4 al 7, se usan las siguientes abreviaturas, gli $=$ glifosato car $=$ carfentrazone; hidro $=$ hidroxal; metsul $=$ metsulfuron metil; sulfato $=$ sulfato de amonio; coad $=$ coadyuvante Nu Film 17 P.

\section{Experimento 2}

Los tratamientos de triclopir + carfentrazone + surfactante (T6), triclopir + carfentrazone + sulfato de amonio + surfactante (T7), triclopir + glifosato + sulfato de amonio + surfactante (T8) y metsulfuron + carfentrazone + surfactante (T12) fueron seleccionados del primer experimento, para ser evaluados, con el fin de discernir si al aplicarlos en diferentes partes de la planta de sainillo, ocurrían diferencias en el control ejercido por éstos.

A partir de la tercera evaluación se encontraron diferencias en el porcentaje del suelo cubierto con sainillo (Cuadro 8). Los tratamientos de triclopir + carfentrazone + surfactante y metsulfuron metil + carfentrazone + surfactante mostraron los menores porcentajes de cobertura.

Cuadro 8. Cobertura del suelo por sainillo (Dieffenbachia oerstedii Schott) (N=9/ tratamiento*), en cuatro épocas. Finca Limofrut C, Matina, Limón, Costa Rica. 2006.

\begin{tabular}{lccccc}
\hline \multirow{2}{*}{ Tratamientos } & \multicolumn{4}{c}{ Épocas de evaluación } \\
\cline { 2 - 6 } & Jul 20** & Ago 3** & Set 13** & Oct 19** & Nov 4** \\
\hline Triclopir + carfentrazone+ coadyuvante & $36,6 \mathrm{a}$ & $12,7 \mathrm{a}$ & $9,7 \mathrm{~b}$ & $22,7 \mathrm{~b}$ & $27,9 \mathrm{~b}$ \\
Triclopir + carfentrazone + sulfato de amonio + coadyuvante & $40,6 \mathrm{a}$ & $13,3 \mathrm{a}$ & $14,2 \mathrm{a}$ & $24,5 \mathrm{~b}$ & $30,6 \mathrm{~b}$ \\
Triclopir + glifosato + sulfato de amonio + coadyuvante & $46,6 \mathrm{a}$ & $18,2 \mathrm{a}$ & $17,7 \mathrm{a}$ & $33,4 \mathrm{a}$ & $44,7 \mathrm{a}$ \\
Metsulsuron metil + carfentrazone + coadyuvante & $54,2 \mathrm{a}$ & $18,8 \mathrm{a}$ & $8,5 \mathrm{~b}$ & $8,7 \mathrm{c}$ & $13,8 \mathrm{c}$ \\
\hline
\end{tabular}

Medias con la misma letra en la misma columna no difieren significativamente, según DMS, $\mathrm{p}<=0,05$.

*Para esta variable no hubo diferencias según el órgano de la planta untado, por lo que las medias que se presentan provienen de nueve datos.

** Datos transformados a arcoseno raíz x. 
En la cuarta y quinta evaluación el tratamiento con el herbicida metsulfuron registró el menor porcentaje de cobertura de sainillo, siendo consistente con los resultados obtenidos en el primer experimento, donde el control por parte de este herbicida fue más lento en comparación con aquellos tratamientos que utilizan triclopir, pero luego se mantuvo por más tiempo.

No se presentaron diferencias significativas en el porcentaje de cobertura del suelo por sainillo entre los tratamientos, respecto al órgano de la planta en donde se untaron (Cuadro 9).

En la cuarta y quinta evaluación se detectaron diferencias en el número de tallos de sainillo con síntomas de fitotoxicidad (Cuadro 10). El tratamiento con el herbicida metsulfuron fue diferente de los otros tratamientos, con el mayor porcentaje de tallos sintomáticos en ambas evaluaciones. Este resultado es consistente con los obtenidos en el primer experimento.

Para el número de tallos de sainillo no se presentaron diferencias significativas entre los tratamientos, respecto al órgano de la planta en donde éstos se untaron (Cuadro 11). En cambio el número de rebrotes del sainillo si se vio influido por el órgano de la arvense que se untó (Cuadros 14 y 15), siendo mayor cuando sólo se untaron las hojas. Este resultado es importante pues sugiere beneficios de untar los tratamientos sobre los tallos, como una mejor alternativa para inhibir rebrotes, que la translocación del herbicida a las yemas del tallo, a partir de las hojas.

En la variable de altura de planta, se presentaron diferencias significativas entre tratamientos hasta la cuarta y quinta evaluación (Cuadro 12). En ambas

Cuadro 9. Estimación de cobertura del suelo por plantas de sainillo (Dieffenbachia oerstedii Schott) en banano, según el órgano de la planta untado $\left(\mathrm{N}=3^{*}\right)$, en cuatro épocas. Finca Limofrut C, Matina, Limón, Costa Rica. 2006.

\begin{tabular}{lccccc}
\hline \multirow{2}{*}{ Tratamientos } & \multicolumn{3}{c}{ Épocas de evaluación } \\
\cline { 2 - 6 } & Jul 20** & Ago 3** & Set 13** & Oct 19** & Nov 4** \\
\hline Toda la planta & $41,7 \mathrm{a}$ & $12,5 \mathrm{a}$ & $10,2 \mathrm{a}$ & $23,4 \mathrm{ab}$ & $27,8 \mathrm{a}$ \\
Sólo a hojas & $47,9 \mathrm{a}$ & $15,8 \mathrm{a}$ & $15,4 \mathrm{a}$ & $25,5 \mathrm{a}$ & $34,1 \mathrm{a}$ \\
Sólo a tallos & $43,8 \mathrm{a}$ & $19,1 \mathrm{a}$ & $11,9 \mathrm{a}$ & $18,1 \mathrm{~b}$ & $25,8 \mathrm{a}$ \\
\hline
\end{tabular}

Medias con la misma letra en la misma columna no difieren significativamente, según DMS, $\mathrm{p}<=0,05$.

* Para cada órgano de la planta, se usaron tres repeticiones por tratamiento herbicida.

** Datos transformados a arcoseno raíz x.

Cuadro 10. Porcentaje de tallos de sainillo (Dieffenbachia oerstedii Schott) sintomáticos (N=9/ tratamiento*) en banano. Finca Limofrut C, Matina, Limón, Costa Rica. 2006.

\begin{tabular}{|c|c|c|c|c|c|}
\hline \multirow{2}{*}{ Tratamientos } & \multicolumn{5}{|c|}{ Épocas de evaluación } \\
\hline & Jul 20** & Ago 3** & Set $13 * *$ & Oct $19 * *$ & Nov $4 * *$ \\
\hline Triclopir + carfentrazone + coadyuvante & 90,0 a & 90,0 a & 69,3 a & $42,3 \mathrm{c}$ & $17,6 \mathrm{~b}$ \\
\hline Triclopir + carfentrazone + sulfato de amonio + coadyuvante & 85,4 a & $88,3 \mathrm{a}$ & $64,5 \mathrm{a}$ & $49,3 \mathrm{~b}$ & $28,2 \mathrm{~b}$ \\
\hline Triclopir + glifosato + sulfato de amonio + coadyuvante & $84,00 \mathrm{a}$ & 85,6 a & 63,8 a & $44,6 \mathrm{bc}$ & $16,1 \mathrm{~b}$ \\
\hline Metsulsuron metil + carfentrazone + coadyuvante & $72,2 \mathrm{a}$ & 87,0 a & 83,0 a & $69,2 \mathrm{a}$ & $53,4 \mathrm{a}$ \\
\hline
\end{tabular}

Medias con la misma letra en la misma columna no difieren significativamente, según DMS, $\mathrm{p}<=0,05$.

* Para esta variable no hubo diferencias según el órgano de la planta untado, por lo que las medias que se presentan provienen de nueve datos.

** Datos transformados a arcoseno raíz $\mathrm{x}$. 
Cuadro 11. Porcentaje de tallos de sainillo (Dieffenbachia oerstedii Schott) sintomáticos en banano, según el órgano de la planta untado (N=3*). Finca Limofrut C. Matina, Limón, Costa Rica. 2006.

\begin{tabular}{llllll}
\hline \multirow{2}{*}{ Tratamientos } & \multicolumn{5}{c}{ Épocas de evaluación } \\
\cline { 2 - 6 } & Jul 20* & Ago 3* & Set 13* & Oct 19* & Nov 4* \\
\hline Toda la planta & $82,3 \mathrm{a}$ & $89,0 \mathrm{a}$ & $73,2 \mathrm{a}$ & $51,3 \mathrm{a}$ & $31,9 \mathrm{a}$ \\
Sólo a hojas & $84,2 \mathrm{a}$ & $86,9 \mathrm{a}$ & $68,8 \mathrm{a}$ & $48,2 \mathrm{a}$ & $23,3 \mathrm{a}$ \\
Sólo a tallos & $82,2 \mathrm{a}$ & $87,3 \mathrm{a}$ & $68,3 \mathrm{a}$ & $54,6 \mathrm{a}$ & $31,3 \mathrm{a}$ \\
\hline
\end{tabular}

Medias con la misma letra en la misma columna no difieren significativamente, según DMS, $\mathrm{p}<=0,05$.

* Para cada órgano de la planta, se usaron tres repeticiones por tratamiento herbicida.

** Datos transformados a arcoseno raíz x.

Cuadro 12. Altura de plantas de sainillo (Dieffenbachia oerstedii Schott) (N=9/ tratamiento*) en banano, en cuatro épocas. Finca Limofrut C. Matina Limón, Costa Rica. 2006.

\begin{tabular}{|c|c|c|c|c|c|}
\hline \multirow{2}{*}{ Tratamientos } & \multicolumn{5}{|c|}{ Épocas de evaluación } \\
\hline & Jul 20** & Ago 3** & Set $13 * *$ & Oct $19 * *$ & Nov $4 * *$ \\
\hline Triclopir + carfentrazone + coadyuvante & $21,4 \mathrm{~b}$ & $14,9 \mathrm{a}$ & $11,2 \mathrm{a}$ & $23,0 \mathrm{~b}$ & $19,9 \mathrm{bc}$ \\
\hline Triclopir + carfentrazone + sulfato de amonio + coadyuvante & $20,8 \mathrm{~b}$ & $15,9 \mathrm{a}$ & $14,9 \mathrm{a}$ & $25,4 \mathrm{~b}$ & $24,3 \mathrm{ab}$ \\
\hline Triclopir + glifosato + sulfato de amonio + coadyuvante & $27,3 \mathrm{~b}$ & $18,1 \mathrm{a}$ & 18,3 a & 34,9 a & $31,0 \mathrm{a}$ \\
\hline metsulsuron metil + carfentrazone + coadyuvante & $45,0 \mathrm{a}$ & $26,1 \mathrm{a}$ & $9,8 \mathrm{a}$ & $6,7 \mathrm{c}$ & $13,9 \mathrm{c}$ \\
\hline
\end{tabular}

* Para la variable altura de plantas de sainillo, no hubo diferencias según el órgano de la planta untado, por lo que las medias que se presentan provienen de nueve datos.

** Medias con la misma letra en la misma columna no difieren significativamente, según DMS, $\mathrm{p}<=0,05$.

evaluaciones el tratamiento que incluía al herbicida metsulfuron, registró la menor altura del sainillo. Esto lo que muestra es la casi total eliminación de la biomasa de la arvense en parcelas tratadas con el metsulfuron.

Al igual que para la variable de rebrote, se presentaron diferencias significativas con respecto al órgano de la planta en donde se untó el tratamiento para la variable altura (Cuadro 13). Esto debido a que al ser untado el tratamiento en el tallo o a toda la planta, con el tiempo los tallos se degradan y disminuye la altura del sainillo, a diferencia de cuando se aplicó sólo a hojas, lo cual permitió que muchos tallos se mantuvieron defoliados pero erguidos (Cuadro 13).
Cuadro 13. Altura de plantas de sainillo (Dieffenbachia oerstedii Schott) en banano, según el órgano de la planta untado $\left(\mathrm{N}=3^{*}\right)$. Finca Limofrut $\mathrm{C}$. Matina, Limón, Costa Rica. 2006.

\begin{tabular}{lccccc}
\hline & \multicolumn{5}{c}{ Épocas de evaluación } \\
\cline { 2 - 6 } Tratamientos & Jul & Ago & Set & Oct & Nov \\
& $\mathbf{2 0}^{* *}$ & $\mathbf{3}^{* *}$ & $\mathbf{1 3}^{* *}$ & $\mathbf{1 9}^{* *}$ & $\mathbf{4}^{* *}$ \\
\hline Toda la planta & $26,3 \mathrm{a}$ & $15,7 \mathrm{a}$ & $11,5 \mathrm{a}$ & $23,8 \mathrm{ab}$ & $20,0 \mathrm{~b}$ \\
Sólo a hojas & $29,1 \mathrm{a}$ & $18,0 \mathrm{a}$ & $17,5 \mathrm{a}$ & $27,1 \mathrm{a}$ & $28,2 \mathrm{a}$ \\
Sólo a tallos & $30,6 \mathrm{a}$ & $22,6 \mathrm{a}$ & $11,7 \mathrm{a}$ & $16,7 \mathrm{a}$ & $18,7 \mathrm{~b}$ \\
\hline
\end{tabular}

* Para cada órgano de la planta, se usaron tres repeticiones por tratamiento herbicida.

** Medias con la misma letra en la misma columna no difieren significativamente, según DMS, $\mathrm{p}<=0,05$. 
Cuadro 14. Rebrote de sainillo (Dieffenbachia oerstedii Schott) $(\mathrm{N}=9 /$ tratamiento*) en banano, en cuatro épocas. Finca Limofrut C. Matina Limón, Costa Rica. 2006.

\begin{tabular}{lrrrrr}
\hline \multirow{2}{*}{ Tratamientos } & \multicolumn{5}{c}{ Épocas de evaluación } \\
\cline { 2 - 6 } & Jul 20** & Ago 3** & Set 13** & Oct 19** & Nov 4** \\
\hline triclopir + carfentrazone + coadyuvante & $0,71 \mathrm{a}$ & $0,92 \mathrm{a}$ & $1,43 \mathrm{a}$ & $2,89 \mathrm{a}$ & $3,63 \mathrm{ab}$ \\
triclopir + carfentrazone + sulfato de amonio + coadyuvante & $0,76 \mathrm{a}$ & $0,82 \mathrm{a}$ & $1,84 \mathrm{a}$ & $2,51 \mathrm{a}$ & $3,1 \mathrm{~b}$ \\
triclopir + glifosato + sulfato de amonio + coadyuvante & $0,88 \mathrm{a}$ & $1,09 \mathrm{a}$ & $1,99 \mathrm{a}$ & $3,01 \mathrm{a}$ & $4,34 \mathrm{a}$ \\
Metsulsuron metil + carfentrazone + coadyuvante & $0,71 \mathrm{a}$ & $1,07 \mathrm{a}$ & $1,03 \mathrm{a}$ & $1,39 \mathrm{~b}$ & $1,95 \mathrm{c}$ \\
\hline
\end{tabular}

* Para esta variable no hubo diferencias según el órgano de la planta untado, por lo que las medias resultaron de nueve datos.

Medias con la misma letra en la misma columna no difieren significativamente, según DMS, $\mathrm{p}<=0,05$.

** Datos transformados a raíz $\mathrm{x}+0,5$.

Cuadro 15. Rebrote de sainillo (Dieffenbachia oerstedii Schott) según el órgano de la planta untado $(\mathrm{N}=3 *)$. Finca Limofrut C. Matina, Limón. Costa Rica. 2006.

\begin{tabular}{lccccc}
\hline Tratamientos & \multicolumn{5}{c}{ Épocas de evaluación } \\
\cline { 2 - 6 } & Jul & Ago & Set & Oct & Nov \\
& $\mathbf{2 0}^{* *}$ & $\mathbf{3}^{* *}$ & $\mathbf{1 3}^{* *}$ & $\mathbf{1 9}^{* *}$ & $\mathbf{4}^{* *}$ \\
\hline Toda la planta & $0,71 \mathrm{a}$ & $0,88 \mathrm{a}$ & $1,32 \mathrm{a}$ & $2,36 \mathrm{a}$ & $3,01 \mathrm{~b}$ \\
Sólo a hojas & $0,79 \mathrm{a}$ & $1,06 \mathrm{a}$ & $1,81 \mathrm{a}$ & $2,76 \mathrm{a}$ & $3,66 \mathrm{a}$ \\
Sólo a tallos & $0,79 \mathrm{a}$ & $1 \mathrm{a}$ & $1,59 \mathrm{a}$ & $2,23 \mathrm{a}$ & $3,1 \mathrm{~b}$ \\
\hline
\end{tabular}

* Datos transformados a raíz $\mathrm{x}+0,5$.

** Medias con la misma letra en la misma columna no difieren significativamente, según DMS, $\mathrm{p}<=0,05$.

Cuadro 16. Corbertura de sainillo (Dieffenbachia oerstedii Schott), en banano. Finca Limofrut C. Matina, Limón, Costa Rica. 2006.

\begin{tabular}{lc}
\hline Tratamientos & Época \\
\cline { 2 - 2 } & Ago 24* \\
\hline $\begin{array}{l}\text { glifosato + coadyuvante }(33: 1 / 66 \% \mathrm{vpc} / \mathrm{v} \\
\text { agua) } \\
\text { glifosato + sulfato de amonio+ coadyuvante }(33:\end{array}$ & $51,7 \mathrm{a}$ \\
$10: 1 \% \mathrm{vpc} / 56 \%$ v agua) & $50,6 \mathrm{ab}$ \\
glifosato + carfentrazone + coadyuvante $(33: 4: 1$ & \\
$\%$ vpc / 62 \% v agua) & $42,8 \mathrm{ab}$ \\
glifosato + carfentrazone + coadyuvante $(33: 8: 1$ & \\
$\%$ vpc / 58 \% v agua) & $31,3 \mathrm{~b}$ \\
glifosato + carfentrazone + coadyuvante $(33: 16:$ & \\
$1 \%$ vpc / 50\% v agua) & $30,2 \mathrm{~b}$ \\
\hline
\end{tabular}

Medias con la misma letra en la misma columna no difieren significativamente, según DMS, $\mathrm{p}<=0,1$.

* Datos transformados a arcoseno raíz x.

\section{Experimento 3}

Los tratamientos de glifosato + carfentrazone, en donde éste último se untó a un volumen del 8 y $16 \%$ (T4 y T5) obtuvieron el menor porcentaje de cobertura y el mayor porcentaje de tallos sintomáticos, siendo diferentes significativamente de los otros tratamientos (Cuadros 16 y 17).

En las variables de altura de plantas de sainillo y rebrotes no se pudo detectar diferencias significativas entre los tratamientos en esta única evaluación (Cuadros 18 y 19). Ocurrió interferencia en algunas prácticas realizadas en la finca sobre las unidades

Cuadro 17. Porcentaje de tallos de sainillo (Dieffenbachia oerstedii Schott) sintomáticos, en banano. Finca Limofrut C. Matina, Limón, Costa Rica. 2006.

\begin{tabular}{|c|c|}
\hline \multirow{2}{*}{ Tratamientos } & Época \\
\hline & Ago 24* \\
\hline $\begin{array}{l}\text { glifosato + coadyuvante }(33: 1 / 66 \% \mathrm{vpc} / \mathrm{v} \\
\text { agua) }\end{array}$ & $29,4 \mathrm{c}$ \\
\hline $\begin{array}{l}\text { glifosato + sulfato de amonio+ coadyuvante ( } 33 \text { : } \\
10: 1 \% \text { vpc / } 56 \% \text { v agua) }\end{array}$ & $27,5 \mathrm{c}$ \\
\hline $\begin{array}{l}\text { glifosato + carfentrazone + coadyuvante }(33: 4: \\
1 \% \text { vpc / } 62 \% \text { v agua })\end{array}$ & $44,3 \mathrm{~b}$ \\
\hline $\begin{array}{l}\text { glifosato + carfentrazone + coadyuvante }(33: 8: \\
1 \% \mathrm{vpc} / 58 \% \text { v agua })\end{array}$ & 70,5 a \\
\hline $\begin{array}{l}\text { glifosato + carfentrazone + coadyuvante }(33: 16: \\
1 \% \text { vpc } / 50 \% \text { v agua })\end{array}$ & $71,3 \mathrm{a}$ \\
\hline
\end{tabular}

Medias con la misma letra en la misma columna no difieren significativamente, según DMS, $\mathrm{p}<=0,05$.

* Datos transformados a arcoseno raíz x. 
Cuadro 18. Altura de plantas de sainillo (Dieffenbachia oerstedii Schott) en banano. Finca Limofrut C. Matina, Limón, Costa Rica. 2006.

\begin{tabular}{|c|c|}
\hline \multirow{2}{*}{ Tratamientos } & Época \\
\hline & Ago 24 \\
\hline $\begin{array}{l}\text { glifosato + coadyuvante }(33: 1 / 66 \% \mathrm{vpc} / \mathrm{v} \\
\text { agua) }\end{array}$ & $54,3 \mathrm{a}$ \\
\hline $\begin{array}{l}\text { glifosato + sulfato de amonio+ coadyuvante ( } 33 \\
: 10: 1 \% \text { vpc / } 56 \% \text { v agua) }\end{array}$ & $45,0 \mathrm{a}$ \\
\hline $\begin{array}{l}\text { glifosato + carfentrazone + coadyuvante }(33: 4 \\
: 1 \% \text { vpc / } 62 \% \text { v agua })\end{array}$ & $50,8 \mathrm{a}$ \\
\hline $\begin{array}{l}\text { glifosato + carfentrazone + coadyuvante }(33: 8 \\
: 1 \% \mathrm{vpc} / 58 \% \mathrm{v} \text { agua })\end{array}$ & $37,3 \mathrm{a}$ \\
\hline $\begin{array}{l}\text { glifosato + carfentrazone + coadyuvante }(33: \\
16: 1 \% \text { vpc / } 50 \% \text { v agua })\end{array}$ & $42,3 \mathrm{a}$ \\
\hline
\end{tabular}

Medias con la misma letra en la misma columna no difieren significativamente, según DMS, $\mathrm{p}<=0,05$.

Cuadro 19. Rebrote de sainillo (Dieffenbachia oerstedii Schott) en banano. Finca Limofrut C. Matina, Limón, Costa Rica. 2006.

\begin{tabular}{lc}
\hline Tratamientos & Evaluación \\
& Ago 24* \\
\hline $\begin{array}{l}\text { glifosato + coadyuvante }(33: 1 / 66 \% \text { vpc / v } \\
\text { agua) } \\
\text { glifosato + sulfato de amonio+ coadyuvante }(33\end{array}$ & $1,41 \mathrm{a}$ \\
$: 10: 1 \% \mathrm{a}$ \\
glifosato + carfentrazone + coadyuvante $(33: 4$ & $1,18 \mathrm{a}$ \\
$: 1 \%$ vpc / 62\% v agua) & \\
glifosato + carfentrazone + coadyuvante $(33: 8$ & $1,22 \mathrm{a}$ \\
$: 1 \%$ vpc/ $58 \%$ v agua $)$ & \\
glifosato + carfentrazone + coadyuvante $(33: 16$ & $0,84 \mathrm{a}$ \\
$: 1 \%$ vpc / 50\% v agua) & \\
\hline
\end{tabular}

Medias con la misma letra en la misma columna no difieren significativamente, según DMS, $\mathrm{p}<=0,05$.

* Datos transformados a raíz $\mathrm{x}+0,5$.

experimentales. Una recaba de los drenajes cercanos a las unidades experimentales, así como la aplicación de cal- dolomita, dañaron parcialmente dichas unidades, por lo que sólo se contó con una evaluación.

En los Cuadros del 20 al 23, se resumen resultados de pruebas de validación en lotes comerciales
Cuadro 20. Porcentaje de suelo cubierto por sainillo (Dieffenbachia oerstedii Schott) en pruebas de validación en áreas comerciales de banano. Finca Limofrut C. Matina, Limón, Costa Rica. 2006.

\begin{tabular}{lllc}
\hline \multirow{2}{*}{ Tratamiento } & \multicolumn{3}{c}{ Épocas de evaluación* } \\
\cline { 2 - 4 } & 34 DDA** & 49 DDA & 79 DDA \\
\hline glifosato + carfentrazone + \\
$\begin{array}{l}\text { coadyuvante }(33: 8: 1 \% \text { vpc } \\
\text { / } 58 \% \text { v agua })\end{array}$ & 12,7 & 17,4 & 14,5 \\
\hline
\end{tabular}

Medias del promedio de seis muestras evaluadas.

* Datos transformados a arcoseno raíz x.

** Días después de la aplicación.

Cuadro 21. Porcentaje de tallos de sainillo (Dieffenbachia oerstedii Schott) sintomáticos, en pruebas de validación en áreas comerciales de banano. Finca Limofrut C. Matina, Limón, Costa Rica. 2006.

\begin{tabular}{lccc}
\hline \multirow{2}{*}{ Tratamiento } & \multicolumn{3}{c}{ Épocas de evaluación* } \\
\cline { 2 - 4 } & 34 DDA** & 49 DDA & 79 DDA \\
\hline glifosato + carfentrazo- & & & \\
ne + coadyuvante $(33$ & 69,7 & 63,9 & 74 \\
$: 8: 1 \%$ vpc / $58 \% \mathrm{v}$ & & & \\
agua) & & & \\
\hline
\end{tabular}

Medias del promedio de seis muestras evaluadas.

* Datos transformados a arcoseno raíz $\mathrm{x}$.

** Días después de la aplicación.

Cuadro 22. Altura $(\mathrm{cm})$ de plantas de sainillo (Dieffenbachia oerstedii Schott) en pruebas de validación en áreas comerciales de banano. Finca Limofrut C. Matina, Limón, Costa Rica. 2006.

\begin{tabular}{lccc}
\hline \multirow{2}{*}{ Tratamiento } & \multicolumn{3}{c}{ Evaluaciones } \\
\cline { 2 - 4 } & 34 DDA* & 49 DDA & 79 DDA \\
\hline glifosato + carfentrazone & & & \\
+ coadyuvante (33:8:1 & 19,7 & 15,8 & 24,5 \\
$\%$ vpc / 58\% v agua) & & & \\
\hline
\end{tabular}

Medias del promedio de seis muestras evaluadas.

* Días después de la aplicación.

con el mejor tratamiento de este estudio. La dosis por hectárea untada en este tratamiento fue de $2.057 \mathrm{~g}$ e. 
Cuadro 23. Número de rebrotes de sainillo (Dieffenbachia oerstedii Schott) en pruebas de validación en áreas comerciales de banano. Finca Limofrut C. Matina, Limón, Costa Rica. 2006.

\begin{tabular}{lccc}
\hline \multirow{2}{*}{ Tratamiento } & \multicolumn{3}{c}{ Épocas de evaluación* } \\
\cline { 2 - 4 } & 34 DDA** & 49 DDA & 79 DDA \\
\hline glifosato + carfentrazone & & & \\
+ coadyuvante $(33: 8: 1$ & 1,4 & 2,4 & 1,3 \\
$\%$ vpc / 58\% v agua) & & & \\
\hline
\end{tabular}

Medias del promedio de seis muestras evaluadas.

* Datos transformados a raíz $\mathrm{x}+0,5$.

** Días después de la aplicación.

a./ha de glifosato $+308,5 \mathrm{~g}$ i. a./ha de carfentrazone, con un porcentaje de cobertura inicial de sainillo de un $40 \%$. Esta dosis puede variar según sea la cobertura de sainillo en un área determinada.

En los tres experimentos y en la etapa de validación, se observó que las plantas tratadas con los tratamientos herbicidas presentaban una serie de lesiones en los tallos de la maleza, semejantes a mordiscos y pudriciones descendentes que no son típicos de los síntomas de fitotoxicidad usualmente asociados a esos herbicidas. Se tomaron muestras de las plantas con los síntomas antes descritos y al realizarles las pruebas de laboratorio se determinó la presencia de bacterias de los géneros Pseudomonas y Erwinia (datos por publicar).

Algunas cepas de esos géneros de bacterias han sido encontradas en el suelo, asociadas a la rizosfera de algunas malezas, donde pueden causar daños en la raíz y son conocidas como "deleterius rhizobacter" (DRB, por sus siglas en inglés) (Rudrappa et al. 2008, Kremer y Kennedy 1996, Kremer et al. 1990). También se encuentran sobre las plantas o en sus alrededores, pero no pueden ingresar a ellas sin que haya una lesión o herida que permita su entrada (Jones et al. 2002, Alfano y Collmer 1996). Una vez dentro de la planta se pueden mover de célula a célula y llegar hasta el xilema y de ahí a toda la planta (Peterson et al. 1981).

Al parecer, los herbicidas utilizados en este estudio causan un estrés y/o una serie de heridas en las plantas de sainillo que permiten el ingreso de estas bacterias, una vez adentro producen una serie de toxinas, polisacáridos extracelulares y enzimas degradadoras de la pared celular que provocan la muerte del tejido vegetal observado en las lesiones descritas (Jones et al. 2002). Se desconoce si en el caso presente, existen otros factores ambientales que pudieron intervenir en el incremento y desarrollo de la infección de estas bacterias en las plantas de sainillo tratadas con los herbicidas utilizados, debido a lo complejo que suelen ser estas interacciones asociadas al estrés en plantas (Schopfer y Brennicke 1999, Johnson et al. 1996).

Por otra parte, se reporta en la literatura que el glifosato puede incrementar la flora microbiana, con aumentos de hasta un $25 \%$ en la biomasa microbiana (Ratcliff et al. 2006). Cabe recalcar que los tratamientos utilizados en la parte de validación tienen a este herbicida dentro de la mezcla de aplicación.

La presencia de las bacterias Erwinia y Pseudomonas en malezas ha sido estudiada (Hoagland 2001, McCarter - Zorner et al. 1985) y algunos autores las proponen como posibles agentes de control biológico (Gronwald et al. 2004, Gronwald et al. 2002, Hoagland 2001, Kremer et al. 1990); en el caso de las DRB, éstas pueden generar una alta selectividad en ciertas plantas, colonizándolas y llegando a ser específicas a su hospedero (Zeller et al. 2007), lo cual incrementaría su potencial como agente de control en un agroecosistema en particular. Sin embargo, el potencial de estas bacterias como agentes de control biológico de arvenses recalcitrantes en el agroecosistema bananero no ha sido estudiado, algo que los autores pretenden hacer a futuro.

\section{CONCLUSIONES}

Los tratamientos del primer experimento que incluyeron al herbicida triclopir (T5, T6, T7 y T8) ejercieron un control rápido sobre sainillo; sin embargo, el rebrote de los tallos que sobrevivieron al untado permitió la recuperación de las poblaciones, en un periodo relativamente corto, lo cual implicaría un mayor número de aplicaciones. Por otra parte, el efecto del triclopir sobre el cultivo del banano es en extremo perjudicial, por lo que no se recomienda su uso en banano. Aún con la técnica de untado con mechero, cuando 
las plantas del sainillo se agovian (menos de 24 horas luego del untado) y, eso coincide con la lluvia, parte del producto puede caer sobre hijos productivos del banano, con daños severos a éstos.

Los tratamientos del primer experimento con el herbicida metsulfuron metil (T11, T12 y T13) obtuvieron al final de las cinco evaluaciones el menor porcentaje de cobertura de sainillo y de rebrotes, las menores alturas de plantas de sainillo, así como el mayor porcentaje de tallos de la maleza sintomáticos, dando el mejor control sobre sainillo en comparación con los otros tratamientos. Se debe tener en cuenta que este herbicida no tiene registro EPA en el cultivo de banano, por lo que su uso podría verse limitado; tampoco es selectivo al cultivo, por lo que debe evitarse su contacto con el banano. Además, en suelos con $\mathrm{pH}$ básicos, su residualidad y movilidad en el suelo pueden resultar inaceptables. Sin embargo, consideramos de interés continuar la investigación con este herbicida, con técnicas de aplicación que eviten el contacto con las plantas de banano. Se debe además estudiar su residualidad en distintos suelos donde se cultiva banano y su potencial absorción por las raíces del cultivo.

Los resultados obtenidos en el segundo experimento fueron consistentes con los del primer experimento. El mejor control sobre sainillo se obtuvo con las mezclas de metsulfuron metil + carfentrazone, en las variables de porcentaje de cobertura y porcentaje de tallos sintomáticos, sin diferencias cuando se untó en tallos, hojas o sobre toda la parte aérea del sainillo.

En las variables de rebrotes y altura de las plantas de sainillo para el tratamiento con metsulfuron metil + carfentrazone + surfactante, se presentaron diferencias con respecto al órgano de la planta en donde fue untado el tratamiento. Lo que indica la importancia de untar toda la parte aérea o los tallos.

La mezcla glifosato + carfentrazone, a las dosis utilizadas en el cuarto y quinto tratamiento en el tercer experimento es promisoria, no sólo por sus efectos sobre el sainillo, sino por cuanto los herbicidas involucrados cuentan con registro EPA para el cultivo de banano. Estos tratamientos en la actualidad se validan a escala comercial.
Es de gran interés concretar un nuevo proyecto de investigación, para el estudio de las otras especies de arvenses recalcitrantes que se asocian al banano. La lista es de 20 especies, aproximadamente.

Las bacterias asociadas a las lesiones en el sainillo, en parcelas aplicadas con varios tratamientos herbicidas, muestran potencial como agentes de control biológico de esta arvense recalcitrante en el agroecosistema bananero.

\section{LITERATURA CITADA}

Alfano, J; Collmer, A. 1996. Bacterial pathogens in plants: Life up against the wall. The Plant Cell 6: 1683-1698.

Arias, E. 1998. Especies de malezas comunes en bananales de Costa Rica. Consorcio Litográfico Herediano. San José. Costa Rica. 96 p.

Croat, T. 1991. Dieffenbachia (Loterías, Dum Cane). In: D. H. Janzen (ed.) Historia natural de Costa Rica. Ed. Universidad de Costa Rica. p 79-81.

Egerer, I. 2005. Ocular involvement due to juice of Dieffenbachia plants (en línea). Alemania. Consultado 8 oct. 2006. Disponible en http://www.ncbi.nlm.nih. gov/entrez/query.fcgi?cmd=Retrieve $\& d b=$ PubMed\&li st_uids $=4999440 \&$ dopt $=$ Abstract

Gronwald, J; Plaisance, K; Ide, D; Wyse, D. 2002. Assessment of Pseudomonas syringae pv. tagetis as a biocontrol agent for Canada thistle. Weed Science, 50: $397-404$.

Gronwald, J; Paisance, K; Baily, B. 2004. Effects of the fungal protein Nep1 and Pseudomonas syringae on growth of Canada thistle (Cirsium arvense), common ragweed (Ambrosia artemisiifolia), and common dandelion (Taraxacum officinale). Weed Science 52: 98-104.

Hammel, B. 2005. Plantas ornamentales nativas de Costa Rica. INBio. Heredia. Costa Rica. 269 p.

Herrera, W. 1985. Clima de Costa Rica: vegetación y clima de Costa Rica. Ed. UNED. Vol 2. San José. Costa Rica. 118 p. 
Hoagland, E. 2001. Symposium: Microbial allelochemicals and pathogens as bioherbicidal agents. Weed Technol. 15: 835-857.

Holdridge, L. 1979. Ecología basada en zonas de vida. San José. Costa Rica. IICA. 216 p.

Johnson, D; Wyse, D; Jones, K. 1996. Controlling weeds with phytopathogenic bacteria. Weed Technol. 10: 621-624

Jones, R; Gruissem, W; Buchanan, B. 2002. Responses to Plant Pathogens. In: Biochemistry \& Molecular Biology of Plants. Wiley \& Sons. Courrier Company Inc. Rockville, USA p. 1102-1154.

Kremer, R; Kennedy, A. 1996. Rhizobacteria as biocontrol agents of weed. Weed Technol. 10: 601-609.

Kremer, R; Begonia, M; Stanley, L; Lanham, E.1990. Characterization of rhizobacteria associated with weed seedlings. Appl. And Envir. Microbiol. 56: 1649-1655.

Kissman, K.; Groth, D. 2000. Plantas infestantes a nocivas. Tomo I. 2 ed. BASF. Sâo Paulo. Brasil. 722 p.

Matysik, A. 2005. Harmful impact of Dieffenbachia sap of the anterior segment of the eye (en línea). Consultado 8 oct. 2006. Disponible en http://www.ncbi.nlm.nih. gov/entrez/query.fcgi?cmd=Retrieve $\& \mathrm{db}=$ PubMed\&li st_uids=4999440\&dopt=Abstract

McCarter-Zorner, N; Harrison, M; Franc, G; Quinn, C; Sells, I; Graham, D. 1985. Soft rot Erwinia bacteria in the rhizospheres of weeds and crop plants in Colorado, United States and Scotland. J.Appl. Bacteriol. 59: 257-368

Nilsson, V; Sánchez, P; Manfredi, R. 2005. Hierbas y arbustos comunes en cafetales y otros cultivos. Universidad Nacional. San José. Costa Rica. 246 p.
Peterson, C; Emanuel, M; Humphreys, G. (1981): Pathway of movement of apoplastic fluorescent dye tracers through the endodermis at the site of secondary root formation in corn and broad bean. Canadian Journal of Botany 59: 618-625.

Prichyd, C; Rudall, P. 1999. Calcium oxalate crystals in monocotyledons: a review of theirs structure and systematics. Annals of Botany 84: 725-739.

Ratcliff, A; Busse, M; Shestak, C. 2006. Changes in microbial community structure following herbicide (glyphosate) additions to forest soils. Applied Soil Ecology 34: 114-124.

Rodríguez, A; Agüero, R. 2000. Identificación de malezas trepadoras del banano (Musa sp.) en la zona Caribe de Costa Rica. Agronomía Mesoamericana 11: 123- 125.

Rudrappa, T; Splaine, R; Biedrzycki, M; Bais, H. 2008. Cyanogenic pseudomonads influence multitrophic interactions in the rhizosphere (en línea). PloS ONE 3(4): 1-11. Consultado 4 ago. 2008. Disponible en: http://www.plosone.org/search/simpleSearch.action?q uery $=$ Cyanogenic + pseudomonads + influence + multitro phic+interactions+in+the+rhizosphere

Schopfer, P; Brennicke, A. 1999. Pflanzer physiologie. Ed. Springler. Berlin - Heidelberg. Alemania. 657 p.

Zeller, S; Brandl, H; Schmid, B. 2007. Host-Plant Selectivity of rhizobacteria in a crop/weed model system. PloS ONE 2(9): 1 - 7. Consultado 4 ago. 2008. Disponible: http://www.plosone.org/article/info\%3Adoi\%2F10.13 71\%2Fjournal.pone.0000846

Zettler, F; Rhodes, B. 1975. Calcium oxalate variability in Dieffenbachia seedlings. HortScience 10(5): 481482. 